hep-ph/9903402

UWThPh-1998-63

HEPHY-PUB 705

March 19, 2018

\title{
Electron and Neutron Electric Dipole Moments in the Constrained MSSM
}

\author{
A. Bartl ${ }^{* 1}$, T. Gajdosik ${ }^{\dagger 2}$, W. Porod ${ }^{\ddagger 1}$, P. Stockinger ${ }^{\S 1}$, and H. Stremnitzer**1 \\ ${ }^{1}$ Institut für Theoretische Physik, Universität Wien, A-1090, Vienna, Austria \\ 2 Institut für Hochenergiephysik der Österreichischen Akademie der Wissenschaften, A-1050, \\ Vienna, Austria
}

\begin{abstract}
We analyze the effects of $C P$-violating phases on the electric dipole moment (EDM) of electron and neutron in the constrained minimal supersymmetric model. We find that the phases $\varphi_{\mu}$ and $\varphi_{A_{0}}$ have to be strongly correlated, in particular for small values of the SUSY mass parameters. We calculate the neutron EDM in two different models, the Quark-Parton Model and the Chiral Quark Model. It turns out that the predictions are quite sensitive to the model used. We show parameter regions in the $M_{0}-M_{1 / 2}$ plane which are excluded by considering simultaneously the experimental bounds of both electron and neutron EDM, assuming specific values for the phases $\varphi_{\mu}$ and $\varphi_{A_{0}}$.
\end{abstract}

11.30.Er, 11.30.Pb, 12.60.Jv, 13.40.Em

Typeset using REVTEX

*email: bartl@ap.univie.ac.at

$\dagger$ email: garfield@hephy . oeaw.ac .at

$\ddagger$ email: porod@ap.univie.ac.at

§email: stocki@hephy. oeaw.ac.at

**email: strem@ap.univie.ac.at 


\section{INTRODUCTION}

The electric dipole moments (EDMs) of electron and neutron are important observables for testing our ideas of $C P$-violation. In the standard model $(\mathrm{SM})$, only one $C P$-violating phase exists in the Cabbibo-Kobayashi-Maskawa matrix. The predictions for the EDMs are extremely small, since the first nonzero contributions arise at two-loop level. They are several orders of magnitude smaller than the experimental limits [1]. Therefore, the EDMs are well suited for testing physics beyond the standard model [2].

In supersymmetric (SUSY) extensions of the standard model, additional $C P$-violating phases are possible. Moreover, the first nonzero contribution to the EDM already shows up at one-loop level. In particular, in the minimal supersymmetric standard model (MSSM) complex parameters can be introduced in the mixing matrices of squarks, sleptons, charginos and neutralinos, therefore yielding more possible sources of $C P$-violation. In weak-scale SUSY, the masses of the lightest supersymmetric particles are expected to be between 100 $\mathrm{GeV}$ and $1 \mathrm{TeV}$. In this case, the EDMs can easily be much larger then the experimental limits and yield constraints on the $C P$-violating phases and on the other parameters of the MSSM. The conclusion would be that either the phases are small or the masses are large [3, 4 . Other arguments like the electroweak origin of the cosmological baryon asymmetry (BAU), however, would favor large $C P$-violating phases with relatively small masses [5]. Therefore, more careful analyses of the supersymmetric contributions to the EDM are necessary to clarify the situation.

A suitable framework for numerical calculations in SUSY is the constrained MSSM, also called minimal supergravity-inspired model (mSUGRA) [6]. In this model universality of the soft SUSY-breaking parameters at the grand unification (GUT) scale is assumed. Parameters of the model are the common gaugino mass $M_{1 / 2}$, the common scalar mass $M_{0}$, the common trilinear scalar coupling parameter $A_{0}$, and $\tan \beta=v_{2} / v_{1}$, with $v_{1,2}$ being the vacuum expectation values of the two Higgs fields. The number of independent complex phases can be reduced to two. The masses at the electroweak scale are determined by using renormalization group equations (RGEs). Such an approach to constrain the phases has recently been used in [7] and [8].

An important aspect in the calculation of the SUSY contributions to the EDMs of electron (eEDM) and neutron (nEDM) is the fact that strong cancellations between the different contributions can occur. This has been particularly emphasized in [9], where the nEDM and eEDM in mSUGRA with two complex phases have been analyzed. Due to this cancellations, the bounds on the phases are less restrictive then those found in previous analyses. Additional constraints on the phases originating from the cosmological bounds on the relic density of neutralinos have been studied in [10]. A different point of view has been presented in [11] where a model with seven independent phases at the electroweak scale has been assumed. Also in this analysis it was found that various cancellations between different contributions occur, and that in large regions of the parameter space the phases are not necessarily small.

In our paper we analyze the eEDM and the nEDM simultaneously in mSUGRA with

complex phases $\varphi_{\mu}$ and $\varphi_{A_{0}}$, which are the phases of the higgsino mass parameter $\mu$ and the trilinear scalar coupling parameter $A_{0}$. We use RGEs to calculate particle masses, couplings, and phases at the electroweak scale from the input parameters at the GUT scale. We confirm the importance of cancellations. We find that quite general the cancellations occur between 
the two most important contributions, which are the chargino and neutralino contribution in the case of the eEDM and the chargino and gluino contribution in the case of the nEDM. Furthermore, the cancellations are only possible if the phases $\varphi_{\mu}$ and $\varphi_{A_{0}}$ are strongly correlated, in particular for small SUSY particle masses. In this case $\varphi_{\mu}$ is strongly restricted. For the nEDM, there is also the problem of evaluating the hadronic matrix element. We use two different approaches, one based on the Quark-Parton Model [12], and a second one based on the Chiral Quark Model [13]. We find that the predictions for the nEDM are very different for the two models used. We show the regions in the $M_{0}-M_{1 / 2}$ plane which are excluded by the experimental bounds for both EDMs for specific values of the phases $\varphi_{\mu}$ and $\varphi_{A_{0}}$. Finally, we also introduce an additional phase $\varphi_{3}$ for the gluino mass parameter and study its influence. We find that also $\varphi_{3}$ is strongly restricted.

In Sec. II we give the expressions for the various contributions for eEDM and the quark EDMs, including the chromoelectric and purely gluonic dimension-six operator. We calculate the nEDM in terms of the quark EDMs in the two different models. In Sec. III we determine the phases and MSSM parameters at the electroweak scale using the RGEs. In Sec. IV we give the numerical analysis of the EDMs within mSUGRA and a discussion of the results. A summary is given in Sec. (V. Explicit forms of the mass matrices for sfermions, charginos, and neutralinos, as well as the expressions for the RGEs are given in the Appendices.

\section{CONTRIBUTIONS TO THE EDM OF ELECTRON AND NEUTRON}

The EDM of a spin- $\frac{1}{2}$ particle is the coefficient $d^{f}$ of the effective operator

$$
\mathcal{L}_{E}=-(i / 2) d^{f} \bar{f} \gamma^{5} \sigma_{\mu \nu} f F^{\mu \nu}
$$

We calculate the supersymmetric contributions to the EDMs of electron and quarks at oneloop level. In the case of the electron EDM we include chargino-sneutrino and neutralinoselectron loops. In the light quark case we include chargino-squark, neutralino-squark, and gluino-squark loops. For the chromoelectric dipole moments of quarks we include charginosquark, neutralino-squark, and gluino-squark loops, whereas the gluonic dimension-six operator gets contributions from loops containing top quark, top squark, and gluino.

The parts of the SUSY Lagrangian that are necessary to calculate the one-loop contributions mentioned above are

$$
\begin{aligned}
\mathcal{L}_{\bar{f} \tilde{\chi}_{k}^{0} \tilde{f}_{m}} & =g \bar{f}\left(a_{m k}^{\tilde{f}} P_{R}+b_{m k}^{\tilde{f}} P_{L}\right) \tilde{\chi}_{k}^{0} \tilde{f}_{m}, \\
\mathcal{L}_{f^{\prime}} \tilde{\chi}_{k}^{+} \tilde{f}_{m} & =g \bar{f}^{\prime}\left(l_{m k}^{\tilde{f}} P_{R}+k_{m k}^{\tilde{f}} P_{L}\right) \tilde{\chi}_{k}^{+} \tilde{f}_{m}, \\
\mathcal{L}_{\bar{q} \tilde{g} \tilde{q}_{m}} & =-\left(g_{s} / \sqrt{2}\right) \bar{q} \lambda^{a}\left(e^{\frac{i}{2} \varphi_{3}} \mathcal{R}_{m 1}^{\tilde{q} *} P_{R}-e^{-\frac{i}{2} \varphi_{3}} \mathcal{R}_{m 2}^{\tilde{q} *} P_{L}\right) \tilde{g}^{a} \tilde{q}_{m}
\end{aligned}
$$

where $g$ and $g_{s}$ are the electroweak and strong coupling constants, respectively, $P_{L, R}=$ $\left(1 \mp \gamma^{5}\right) / 2, a=1 \ldots 8$ are the gluino color indices, $\lambda^{a}$ are the Gell-Mann matrices, $\varphi_{3}$ is the phase of the soft-breaking gluino mass. To simplify the notation the quark and squark

color indices are suppressed. The scalar fields $\tilde{f}_{L}$ and $\tilde{f}_{R}$ are linear combinations of the mass eigenstates $\tilde{f}_{1,2}$ : 


$$
\left(\begin{array}{l}
\tilde{f}_{1} \\
\tilde{f}_{2}
\end{array}\right)=\mathcal{R}^{\tilde{f}}\left(\begin{array}{l}
\tilde{f}_{L} \\
\tilde{f}_{R}
\end{array}\right),
$$

where $\mathcal{R}^{\tilde{f}}$ is the unitary diagonalization matrix defined in Eq. (A8). Note that $\mathcal{R}^{\tilde{f}}$ depends on the phases $\varphi_{\mu}$ and $\varphi_{A_{0}}$ via the off diagonal entry of the squark mass matrix, see Eq. (A5) and Table $[$. The couplings are defined as (we use the notation of [14]):

$$
\begin{aligned}
& l_{m j}^{\tilde{\nu}}=-\delta_{m 1} V_{j 1}, \\
& l_{m j}^{\tilde{u}}=-\mathcal{R}_{m 1}^{\tilde{u} *} V_{j 1}+Y_{u} \mathcal{R}_{m 2}^{\tilde{u} *} V_{j 2}, \\
& l_{m j}^{\tilde{e}, \tilde{d}}=-\mathcal{R}_{m 1}^{\tilde{e}, \tilde{d} *} U_{j 1}+Y_{e, d} \mathcal{R}_{m 2}^{\tilde{e}, \tilde{d} *} U_{j 2}, \\
& k_{m j}^{\tilde{\nu}}=Y_{e} \delta_{m 1} U_{j 2}^{*}, \\
& k_{m j}^{\tilde{e}}=0 \text {, } \\
& k_{m j}^{\tilde{u}}=Y_{d} \mathcal{R}_{m 1}^{\tilde{u} *} U_{j 2}^{*}, \\
& k_{m j}^{\tilde{d}}=Y_{u} \mathcal{R}_{m 1}^{\tilde{d} *} V_{j 2}^{*}, \\
& a_{m j}^{\tilde{f}}=\mathcal{R}_{m 1}^{\tilde{f} *} f_{L j}^{\tilde{f}}+\mathcal{R}_{m 2}^{\tilde{f} *} h_{R j}^{\tilde{f}}, \\
& b_{m j}^{\tilde{f}}=\mathcal{R}_{m 1}^{\tilde{f} *} h_{L j}^{\tilde{f}}+\mathcal{R}_{m 2}^{\tilde{f} *} f_{R j}^{\tilde{f}}, \\
& h_{L j}^{u}=Y_{u}\left(\sin \beta N_{3 j}-\cos \beta N_{4 j}\right) \text {, } \\
& h_{R j}^{u}=Y_{u}\left(\sin \beta N_{3 j}^{*}-\cos \beta N_{4 j}^{*}\right)=h_{L j}^{u *} \text {, } \\
& h_{L j}^{e, d}=-Y_{e, d}\left(\cos \beta N_{3 j}+\sin \beta N_{4 j}\right) \text {, } \\
& h_{R j}^{e, d}=-Y_{e, d}\left(\cos \beta N_{3 j}^{*}+\sin \beta N_{4 j}^{*}\right)=h_{L j}^{e, d *}, \\
& f_{L j}^{f}=-\left[Q_{f} \sin 2 \theta_{W} N_{1 j}^{*}+\left(1-2 Q_{f} \sin ^{2} \theta_{W}\right) N_{2 j}^{*}\right] /\left(\sqrt{2} \cos \theta_{W}\right), \\
& f_{R j}^{f}=\left[Q_{f} \sin 2 \theta_{W} N_{1 j}+\left(-2 Q_{f} \sin ^{2} \theta_{W}\right) N_{2 j}\right] /\left(\sqrt{2} \cos \theta_{W}\right), \\
& Y_{u}=\frac{m_{u}}{\sqrt{2} m_{W} \sin \beta}, \\
& Y_{e, d}=\frac{m_{e, d}}{\sqrt{2} m_{W} \cos \beta} .
\end{aligned}
$$

$Q_{f}$ and $Y_{f}$ are electric and Yukawa couplings of the fermion $f, \theta_{W}$ is the Weinberg angle, and $\tan \beta=v_{2} / v_{1}$ is the ratio of the Higgs vacuum expectation values $v_{1}$ and $v_{2}$. $U$ and $V$ 
are the unitary matrices which diagonalize the chargino mass matrix, Eq. (B1). $N_{\alpha j}$ is the unitary matrix which diagonalizes the complex symmetric neutralino mass matrix, Eq. (C3). For diagonalizing we use the singular value decomposition.

A generic form for the one-loop EDM of spin-1/2 particles due to exchange of fermions and scalar particles has been worked out in [15]. Extensions of the EDMs to the full electric and weak dipole moment form factors for the top quark have been given in [16]. A nonvaninshing EDM demands a change in chirality of the external fermion and involves the imaginary parts of the couplings. In the following we give the complete analytic expressions for the individual one-loop contributions. We have compared our results with 9, 11] and found agreement.

\section{A. Chargino Contribution}

The chargino contribution to the EDM of the fermion $f$ is given by

$$
\frac{1}{e} d_{\tilde{\chi}^{+}}^{f}=\frac{\alpha}{4 \pi \sin ^{2} \theta_{W}} \sum_{m, j=1}^{2} \operatorname{Im}\left[\Gamma_{m j}^{f}\right] \frac{m_{\tilde{\chi}_{j}^{+}}}{m_{\tilde{f}_{m}^{\prime}}^{2}}\left(Q_{f^{\prime}} B\left(\frac{m_{\tilde{\chi}_{j}^{+}}^{2}}{m_{\tilde{f}_{m}^{\prime}}^{2}}\right)+\left(Q_{f}-Q_{f^{\prime}}\right) A\left(\frac{m_{\tilde{\chi}_{j}^{+}}^{2}}{m_{\tilde{f}_{m}^{\prime}}^{2}}\right)\right),
$$

where $\alpha=e^{2} /(4 \pi)$ and $e=g \sin \theta_{W} \cdot f^{\prime}$ is the isospin partner of $f$ in the $\mathrm{SU}(2)$-doublet. Neglecting the mass of the external fermions (in our case electron, up, down, and strange quark) the functions $\mathrm{A}$ and $\mathrm{B}$ have the simple form 15

$$
\begin{aligned}
& A(r)=\frac{1}{2(1-r)^{2}}\left(3-r+\frac{2 \ln r}{1-r}\right), \\
& B(r)=\frac{1}{2(1-r)^{2}}\left(1+r+\frac{2 r \ln r}{1-r}\right) .
\end{aligned}
$$

The first and second term in Eq. (2.12) are due to the Feynman diagrams Fig. 1a and Fig. 1b, respectively. The expressions $\operatorname{Im}\left[\Gamma_{m j}^{f}\right]$ are given by:

$$
\begin{gathered}
\operatorname{Im}\left[\Gamma_{m j}^{e}\right]=\operatorname{Im}\left[Y_{e} U_{j 2} V_{j 1}\right] \delta_{m 1} \\
\operatorname{Im}\left[\Gamma_{m j}^{u}\right]=\operatorname{Im}\left[Y_{u} V_{j 2} \mathcal{R}_{m 1}^{\tilde{d}}\left(U_{j 1} \mathcal{R}_{m 1}^{\tilde{d} *}-Y_{d} U_{j 2} \mathcal{R}_{m 2}^{\tilde{d} *}\right)\right] \\
=(1 / 2) Y_{u}\left(\left(1-(-1)^{m} \cos 2 \theta_{\tilde{d}}\right) \operatorname{Im}\left[U_{j 1} V_{j 2}\right]+Y_{d}(-1)^{m} \sin 2 \theta_{\tilde{d}} \operatorname{Im}\left[U_{j 2} V_{j 2} e^{i \varphi_{\tilde{d}}}\right]\right),
\end{gathered}
$$

and

$$
\begin{aligned}
\operatorname{Im}\left[\Gamma_{m j}^{d}\right] & =\operatorname{Im}\left[Y_{d} U_{j 2} \mathcal{R}_{m 1}^{\tilde{u}}\left(V_{j 1} \mathcal{R}_{m 1}^{\tilde{u} *}-Y_{u} V_{j 2} \mathcal{R}_{m 2}^{\tilde{u} *}\right)\right] \\
& =(1 / 2) Y_{d}\left(\left(1-(-1)^{m} \cos 2 \theta_{\tilde{u}}\right) \operatorname{Im}\left[U_{j 2} V_{j 1}\right]+Y_{u}(-1)^{m} \sin 2 \theta_{\tilde{u}} \operatorname{Im}\left[U_{j 2} V_{j 2} e^{i \varphi_{\tilde{u}}}\right]\right)
\end{aligned}
$$




\section{B. Neutralino Contribution}

The neutralino contribution to the fermion EDM is given by

$$
\frac{1}{e} d_{\tilde{\chi}^{0}}^{f}=-\frac{Q_{f}}{8 \pi} \frac{\alpha}{\sin ^{2} \theta_{W}} \sum_{k=1}^{4} \sum_{m=1}^{2} \eta_{m k}^{f} \frac{m_{\tilde{\chi}_{k}^{0}}}{m_{\tilde{f}_{m}}^{2}} B\left(\frac{m_{\tilde{\chi}_{k}^{0}}^{2}}{m_{\tilde{f}_{m}}^{2}}\right)
$$

where

$$
\begin{aligned}
\eta_{m k}^{f}= & (-1)^{m} \sin 2 \theta_{\tilde{f}} \operatorname{Im}\left[\left(\left(h_{L k}^{f}\right)^{2}-f_{L k}^{f} f_{R k}^{f *}\right) e^{-i \varphi_{\tilde{f}}}\right] \\
& -\left(1-(-1)^{m} \cos 2 \theta_{\tilde{f}}\right) \operatorname{Im}\left[h_{L k}^{f} f_{L k}^{f *}\right] \\
& -\left(1+(-1)^{m} \cos 2 \theta_{\tilde{f}}\right) \operatorname{Im}\left[h_{L k}^{f} f_{R k}^{f}\right] .
\end{aligned}
$$

\section{Gluino Contribution}

The gluino contribution to the quark EDM is given by

$$
\begin{aligned}
\frac{1}{e} d_{\tilde{g}}^{q} & =-\frac{2 \alpha_{s}}{3 \pi} \sum_{k=1}^{2} \operatorname{Im}\left[e^{i \varphi_{3}} \mathcal{R}_{k 2}^{\tilde{q}} \mathcal{R}_{k 1}^{\tilde{q} *}\right] \frac{m_{\tilde{g}}}{m_{\tilde{q}_{k}}^{2}} Q_{q} B\left(\frac{m_{\tilde{g}}^{2}}{m_{\tilde{q}_{k}}^{2}}\right) \\
& =\frac{\alpha_{s}}{3 \pi} \sin \left(\varphi_{3}-\varphi_{\tilde{q}}\right) \sin 2 \theta_{\tilde{q}} \sum_{k=1}^{2}(-1)^{k} \frac{m_{\tilde{g}}}{m_{\tilde{q}_{k}}^{2}} Q_{q} B\left(\frac{m_{\tilde{g}}^{2}}{m_{\tilde{q}_{k}}^{2}}\right)
\end{aligned}
$$

where $\alpha_{s}=g_{s}^{2} / 4 \pi$ and $m_{\tilde{g}}$ is the gluino mass.

\section{Quark Chromoelectric Dipole Moment and Gluonic Dimension-Six Operator}

The quark chromoelectric dipole moment is defined as the coefficient $\hat{d}^{q}$ in the effective operator

$$
\mathcal{L}_{C}=-(i / 2) \hat{d}^{q} \bar{q} \sigma_{\mu \nu} \gamma^{5}\left(\lambda^{a} / 2\right) q G^{a \mu \nu}
$$

The chromoelectric dipole moment has also chargino, neutralino, and gluino contributions. They are given by [9]

$$
\begin{gathered}
\hat{d}_{\tilde{\chi}^{+}}^{q}=-\frac{g_{s} \alpha}{4 \pi \sin ^{2} \theta_{W}} \sum_{m, j=1}^{2} \operatorname{Im}\left[\Gamma_{m j}^{q}\right] \frac{m_{\tilde{\chi}_{j}^{+}}}{m_{\tilde{q}_{m}}^{2}} B\left(\frac{m_{\tilde{\chi}_{j}^{+}}}{m_{\tilde{q}_{m}}^{2}}\right), \\
\hat{d}_{\tilde{\chi}^{0}}^{q}=-\frac{g_{s} \alpha}{8 \pi \sin ^{2} \theta_{W}} \sum_{m=1}^{2} \sum_{k=1}^{4} \eta_{m k}^{q} \frac{m_{\tilde{\chi}_{k}^{0}}}{m_{\tilde{q}_{m}}^{2}} B\left(\frac{m_{\tilde{\chi}_{k}^{0}}}{m_{\tilde{q}_{m}}^{2}}\right),
\end{gathered}
$$

and 


$$
\begin{aligned}
\hat{d}_{\tilde{g}}^{q} & =-\frac{g_{s} \alpha_{s}}{4 \pi} \sum_{k=1}^{2} \operatorname{Im}\left[e^{i \varphi_{3}} \mathcal{R}_{k 2}^{\tilde{q}} \mathcal{R}_{k 1}^{\tilde{q} *}\right] \frac{m_{\tilde{g}}}{m_{\tilde{q}_{k}}^{2}} C\left(\frac{m_{\tilde{g}}^{2}}{m_{\tilde{q}_{k}}^{2}}\right) \\
& =\frac{g_{s} \alpha_{s}}{8 \pi} \sin \left(\varphi_{3}-\varphi_{\tilde{q}}\right) \sin 2 \theta_{\tilde{q}} \sum_{k=1}^{2}(-1)^{k} \frac{m_{\tilde{g}}}{m_{\tilde{q}_{k}}^{2}} C\left(\frac{m_{\tilde{g}}^{2}}{m_{\tilde{q}_{k}}^{2}}\right)
\end{aligned}
$$

where

$$
C(r)=3 A(r)-(1 / 3) B(r)
$$

The Wilson coefficient $d_{G}$ of the $C P$-violating gluonic dimension-six operator is defined through

$$
\mathcal{L}_{G}=-(1 / 6) d_{G} G_{\mu \nu a} G_{b}^{\nu \rho} \tilde{G}_{\rho c}^{\mu} f_{a b c} .
$$

The leading nontrivial contribution to $d_{G}$ in the MSSM is given by a two-loop diagram involving top, scalar top, and gluino [9,17]:

$$
d_{G}=\frac{3 \alpha_{s}^{2} g_{s} m_{t}}{32 \pi^{2}} \sin \varphi_{\tilde{t}} \sin 2 \theta_{\tilde{t}} \frac{m_{\tilde{t}_{1}}^{2}-m_{\tilde{t}_{2}}^{2}}{m_{\tilde{g}}^{5}} H\left(\frac{m_{\tilde{t}_{1}}^{2}}{m_{\tilde{g}}^{2}}, \frac{m_{\tilde{t}_{2}}^{2}}{m_{\tilde{g}}^{2}}, \frac{m_{t}^{2}}{m_{\tilde{g}}^{2}}\right) .
$$

The definition of the two-loop function $H$ can be found in [17].

\section{E. EDM of electron and neutron}

Having defined the contributions from the individual Feynman diagrams, we can now write down the total EDM of the electron as the sum of neutralino and chargino contributions:

$$
d^{e}=d_{\tilde{\chi}^{+}}^{e}+d_{\tilde{\chi}^{0}}^{e}
$$

In order to obtain the EDM of the neutron in terms of the quark EDMs, a specific description of the neutron as quark bound state is needed. Throughout this paper we use two different approaches.

1.) The relativistic Quark-Parton Model: In this model, the contributions of the quarks to the $\mathrm{nEDM}$ are given in terms of quantities $\Delta_{q}$ [12], which are measured in polarized lepton-nucleon scattering:

$$
d^{n}=\eta^{E}\left(\Delta_{u} d^{d}+\Delta_{d} d^{u}+\Delta_{s} d^{s}\right)
$$

where the individual quark contributions are again given in terms of chargino, neutralino, and gluino contributions

$$
d^{q}=d_{\tilde{\chi}^{+}}^{q}+d_{\tilde{\chi}^{0}}^{q}+d_{\tilde{g}}^{q} .
$$

As already stated, the $\Delta_{q}$ are the measured contributions of the quark $q$ to the spin of the

proton; to use them for the neutron we have taken advantage of a simple isospin relation. For 
definiteness we use the values given in Ref. [18]: $\Delta_{u}=0.746, \Delta_{d}=-0.508$, and $\Delta_{s}=-0.226$. The QCD correction factor $\eta^{E}$ takes into account that the quark EDM analysis is done at the electroweak scale and hence has to be evolved down to the hadronic scale with the help of RGEs. We use $\eta^{E}=1.53$ as given in Ref. [19.

2.) The Chiral Quark Model: This model is based on the effective chiral quark theory given in Ref. [13]. The contribution of the quark EDMs to the nEDM is given by the nonrelativistic $S U(6)$ coefficients

$$
d^{n}=(4 / 3) d^{d}-(1 / 3) d^{u} .
$$

The quark EDMs in this model are given by contributions of all quark and gluon operators (to leading order in $\alpha_{s}$ ) with the proper dimensional rescaling. This yields

$$
d^{q}=\eta^{E}\left(d_{\tilde{\chi}^{+}}^{q}+d_{\tilde{\chi}^{0}}^{q}+d_{\tilde{g}}^{q}\right)+\eta^{C} \frac{e}{4 \pi}\left(\hat{d}_{\tilde{\chi}^{+}}^{q}+\hat{d}_{\tilde{\chi}^{0}}^{q}+\hat{d}_{\tilde{g}^{q}}^{q}\right)+\eta^{G} \frac{e \Lambda_{S B}}{4 \pi} d_{G} .
$$

$\eta^{E}, \eta^{C}$, and $\eta^{G}$ are the QCD correction factors due to RGEs, whereas $\Lambda_{S B}$ is the scale of chiral symmetry breaking in QCD; we use $\eta^{E}=1.53$ [19], $\eta^{C} \simeq \eta^{G} \simeq 3.4$ (as used in [9]), and $\Lambda_{S B} \simeq 1.19 \mathrm{GeV}[13]$.

\section{DETERMINATION OF THE MSSM PARAMETERS AND PHASES}

The formulas for the EDMs, when evaluated in the MSSM with complex parameters in its most general form, contain too many free parameters. In order to study the constraints of the EDMs on the phases and mass parameters we have to reduce the number of free parameters by further theoretical assumptions. Therefore, we assume universality conditions for gaugino, sfermion, and Higgs mass parameters and the trilinear couplings

$$
\begin{aligned}
M_{0} & :=M_{\tilde{E}_{i}}=M_{\tilde{L}_{i}}=M_{\tilde{D}_{i}}=M_{\tilde{Q}_{i}}=M_{\tilde{U}_{i}}=m_{H_{1}}=m_{H_{2}}, \\
M_{1 / 2} & :=M_{1}=M_{2}=M_{3}, \\
A_{0} & :=A_{e_{i}}=A_{d_{i}}=A_{u_{i}}
\end{aligned}
$$

at the GUT scale $M_{G U T}$ [6], where $i=1,2,3$ is the generation index. We determine the parameters at the electroweak scale with the help of the RGEs as given in [20].

At the electroweak scale the following parameters can be complex: the trilinear couplings $A_{f_{i}}$, the gaugino mass parameters $M_{k}$, and the Higgs parameters $\mu$ and $B$. The product $\mu B$ and the gaugino mass parameter $M_{2}$ can be made real by redefinition of the fields. $|\mu|$ and $B$ are determined by requiring the correct electroweak symmetry breaking:

$$
\begin{aligned}
|\mu|^{2} & =\frac{\left(m_{H_{1}}^{2}+\Delta T_{1}\right)-\left(m_{H_{2}}^{2}+\Delta T_{2}\right) \tan ^{2} \beta}{\tan ^{2} \beta-1}-\frac{1}{2} m_{Z}^{2}, \\
2 \mu B & =\left(m_{H_{1}}^{2}+m_{H_{2}}^{2}+2|\mu|^{2}+\Delta T_{1}+\Delta T_{2}\right) \sin 2 \beta,
\end{aligned}
$$

where $\Delta T_{1,2}$ denote the leading one-loop corrections to the tadpole equations stemming from top, scalar top, bottom, and sbottom contributions 20 22. The phase of $\mu, \varphi_{\mu}$, remains

a free parameter. $\varphi_{\mu}$ can be specified at any scale, because it does not evolve with the 
corresponding RGE up to two loops [23]. In order to determine the phases at the electroweak scale we assume $M_{1 / 2}$ real, and $A_{0}$ and $\mu$ complex at the GUT scale. Note that at one-loop level only the phase difference between the phases of $A_{0}$ and $M_{1 / 2}$ is physically relevant. We summarize the complex phases entering the mass matrices in Table [.

We use the following procedure for determining the soft SUSY-breaking parameters at the electroweak scale. We specify the gauge couplings, $\tan \beta$, and the Yukawa couplings of the third generation at the electroweak scale. We take $A_{0}, M_{0}, M_{1 / 2}$ at $M_{G U T}$ with Eqs. (3.1)(3.3) as boundary conditions. The RGEs are given in the $\overline{\mathrm{DR}}$ scheme. We evolve the RGEs for the gauge couplings at two-loop level from $Q=m_{Z}$ to $Q=M_{G U T}$ which is determined by the condition $g_{1}=g_{2}$. We evolve the RGEs for the Yukawa couplings at the one-loop level, because they enter the RGEs of the gauge couplings at two-loop level. We take into account threshold effects by including step functions for the coefficients of the beta functions (see e.g. [20]). For simplicity we assume that there is no mixing between the generations. We then evolve the RGEs for the soft SUSY-breaking parameters from $M_{G U T}$ to $m_{Z}$. The mass parameters $M_{j}$ are decoupled from the RGEs if $M_{j}(Q)=Q$ is satisfied. We calculate $|\mu|$ and $B$ by requiring correct electroweak symmetry breaking Eqs. (3.4) and (3.5). The corrections are sensitive to the relative phases between the $A$ parameters and $\mu$. This phase dependence may change $|\mu|$ by a few $\mathrm{GeV}$, which is in the range of the error expected by neglecting the other contributions to the one-loop corrected tadpoles [21,22]. We iterate the complete procedure until the parameters vary less than $1 \%$.

For the discussion in the next Section it is convenient to have the following approximations for the parameters at the electroweak scale at hand (the exact formulas for the one-loop results are given in Appendix D). With $\alpha_{G U T}=1 / 24$ and $M_{G U T}=2.38 \times 10^{16} \mathrm{GeV}$ we get:

$$
\begin{aligned}
& M_{\tilde{L}}^{2} \simeq M_{0}^{2}+0.52 M_{1 / 2}^{2}, \\
& M_{\tilde{E}}^{2} \simeq M_{0}^{2}+0.15 M_{1 / 2}^{2}, \\
& M_{\tilde{Q}}^{2} \simeq M_{0}^{2}+6.7 M_{1 / 2}^{2}, \\
& M_{\tilde{U}}^{2} \simeq M_{\tilde{D}}^{2} \simeq M_{0}^{2}+6.2 M_{1 / 2}^{2}, \\
& M_{1}=M^{\prime} \simeq 0.41 M_{1 / 2}, \\
& M_{2}=M \simeq 0.82 M_{1 / 2}, \\
& \quad M_{3} \simeq 2.82 M_{1 / 2}, \\
& \\
& A_{t} \simeq(1-y) A_{0}-2 M_{1 / 2}, \\
& A_{u} \simeq\left(1-\frac{y}{2}\right) A_{0}-2.8 M_{1 / 2}, \\
& A_{d} \simeq A_{0}-3.6 M_{1 / 2}, \\
& A_{e} \simeq A_{0}-0.7 M_{1 / 2},
\end{aligned}
$$

where y varies between 0.85 and 1 for $40>\tan \beta>1$. Eqs. (3.6a)-(3.6d) are only valid for the first and second generation. Note that Eqs. (3.8a) -(3.8d) have strong implications for the $A$ parameters at the electroweak scale. If one takes, for example, $M_{1 / 2}$ real and $A_{0}$

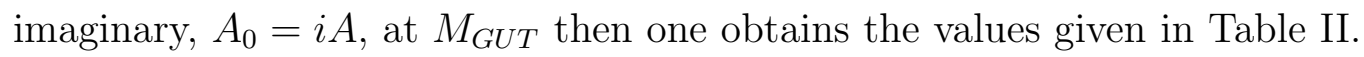




\section{EDM ANALYSIS WITHIN MSUGRA}

In this Section we investigate the EDM of electron and neutron in the framework of mSUGRA with complex parameters. As outlined in Sec. [1], this model is completely specified by six parameters: $M_{0}, M_{1 / 2},\left|A_{0}\right|, \tan \beta$ and the phases $\varphi_{A_{0}}$ and $\varphi_{\mu}$. The experimental bounds obtained in [1] are $\left|d^{e}\right| \leq d_{\exp }^{e}=4.3 \times 10^{-27} e \mathrm{~cm}$ and $\left|d^{n}\right| \leq d_{\exp }^{n}=1.1 \times 10^{-25} e \mathrm{~cm}$.

For the eEDM we have two supersymmetric contributions stemming from neutralino and chargino exchange, Figs. $1 \mathrm{a}$ and 1b, respectively. The chargino contribution depends explicitly on the phase $\varphi_{\mu}$, the dependence on $\varphi_{A_{0}}$ comes only through the RGEs and is very weak. The neutralino contribution depends explicitly on $\varphi_{\mu}$ and $\varphi_{A_{0}}$. In the major part of the parameter space the chargino contribution dominates. The reasons are: (i) The loop function $A(r)$, Eq. (2.13), entering in the chargino contribution, is larger than $B(r)$, Eq. (2.14), which enters the neutralino contribution. (ii) The neutralino contribution is proportional to the selectron mixing angle $\sin 2 \theta_{\tilde{e}}$, which is usually rather small.

In Fig. 2 we show $d_{\tilde{\chi}^{0}}^{e}$, the neutralino contribution of the eEDM, as a function of the $C P$-violating phases $\varphi_{\mu}$ and $\varphi_{A_{0}}$ with the other parameters fixed: $M_{0}=150 \mathrm{GeV}, M_{1 / 2}=$ $200 \mathrm{GeV},\left|A_{0}\right|=450 \mathrm{GeV}$, and $\tan \beta=3$. As can be seen, the neutralino contribution alone already exceeds the experimental limit. The calculated eEDM is below the experimental limit only if cancellations between chargino and neutralino contributions occur. In this case the eEDM depends significantly on the phase $\varphi_{A_{0}}$ if either $\left|\varphi_{\mu}\right| \ll\left|\varphi_{A_{0}}\right|$ or $\left|A_{e}\right| \gtrsim|\mu| \tan \beta$. In the first case the chargino contribution is small because it is proportional to $\sin \varphi_{\mu}$, therefore, the neutralino contribution can be of the same order of magnitude as the chargino contribution. In the second case the relevant phase in the neutralino contribution is determined by the off-diagonal element of the selectron mixing matrix Eq. (A5). In the mSUGRA model the absolute value of $\mu$ is fixed by the condition of radiative electroweak symmetry breaking Eq. (3.4). It turns out that $|\mu|$ has always roughly the same order of magnitude as $\left|A_{e}\right|$ in the parameter region considered. Note that the neutralino contribution depends not only on the phase of $\left(A_{e}-\mu^{*} \tan \beta\right)$, Eq. (A5), but also directly on $\varphi_{\mu}$ via the neutralino mixing matrix, as can be seen in Eqs. (2.19), (2.9d)-(2.11b).

Due to the cancellation mechanism between chargino and neutralino contribution it is not straightforward to conclude which mSUGRA parameter values and phases are excluded by the experimental upper bound of the eEDM. To answer this question we show in Fig. 3 the regions in the $M_{0}-M_{1 / 2}$ plane that are allowed by the experimental limit on the eEDM for different values of the phase $\varphi_{\mu}$. In doing so we have taken $\varphi_{A_{0}}=\pi / 2$, which is the maximal phase difference between $M_{1 / 2}$ and $A_{0}$ at the GUT scale, $\tan \beta=3$, and $\left|A_{0}\right|=3 M_{0}$. For example, choosing $\varphi_{\mu}=-0.1$, the region in the $M_{0}-M_{1 / 2}$ plane to the left of the dasheddotted line is excluded. As can be seen, the parameters $M_{0}=120 \mathrm{GeV}$ and $M_{1 / 2}=160 \mathrm{GeV}$ are allowed and give relatively light SUSY particle masses (for illustration: $m_{\tilde{\chi}_{1}^{0}}=58 \mathrm{GeV}$, $\left.m_{\tilde{\chi}_{1}^{ \pm}}=106 \mathrm{GeV}, m_{\tilde{\nu}_{e}}=157 \mathrm{GeV}, m_{\tilde{e}_{1}}=160 \mathrm{GeV}, m_{\tilde{e}_{2}}=163 \mathrm{GeV}\right)$. Taking $\varphi_{\mu}=-0.54$, only values of $\left(M_{1 / 2}, M_{0}\right)$ to the right of the solid line are allowed which, for example, means $M_{1 / 2} \gtrsim 0.9 \mathrm{TeV}$ if $M_{0}=1.5 \mathrm{TeV}$ or $M_{1 / 2} \gtrsim 1.5 \mathrm{TeV}$ if $M_{0}=0.7 \mathrm{TeV}$. In this case rather heavy SUSY particles are predicted, (i. e. $m_{\tilde{\chi}_{1}^{0}}>398 \mathrm{GeV}, m_{\tilde{\chi}_{1}^{ \pm}}>764 \mathrm{GeV}$, $\left.m_{\tilde{\nu}_{e}}>1170 \mathrm{GeV}, m_{\tilde{e}_{1}}>1073 \mathrm{GeV}, m_{\tilde{e}_{2}}>1171 \mathrm{GeV}\right)$. The bending in the dotted line for $\varphi_{\mu}=-0.18$ is caused by the cancellation mechanism between chargino and neutralino 
contributions. The grey area is excluded, because the condition of radiative electroweak symmetry breaking is not fulfilled.

Up to now we have only considered the eEDM. Now we consider the eEDM and the nEDM simultanously. Taking into account also the experimental upper limit on the nEDM will enlarge the excluded parameter region. The predicted value for the nEDM depends strongly on the neutron model which relates the nEDM to the EDM of its constituents. To demonstrate this fact we calculate the nEDM in the Quark-Parton Model and in the Chiral Quark Model as described in Sec. IIE. Also for the nEDM to fulfill the experimental bounds it is necessary that strong cancellations between the different contributions occur.

Another way to show the systematics of these cancellations is to plot the allowed region in the $\varphi_{\mu}-\varphi_{A_{0}}$ plane. In the Figs. 4, 5, and 8 we consider rather small mSUGRA parameters: $M_{0}=150 \mathrm{GeV}, M_{1 / 2}=200 \mathrm{GeV},\left|A_{0}\right|=450 \mathrm{GeV}$, and $\tan \beta=3$. In all $\varphi_{\mu^{-}} \varphi_{A_{0}}$ plots (Figs. 4, 7, and 8), the allowed values of the phases are within the small bands between the lines. In Figs. 4, 6, 7, and 8 we discuss the eEDM together with the nEDM. As can be seen from the dotted lines for the allowed region of the eEDM in Figs. $4 \mathrm{a}$ and $4 \mathrm{~b}, \varphi_{\mu}$ is bounded, $\left|\varphi_{\mu}\right| \lesssim 0.1$, whereas $\varphi_{A_{0}}$ is essentially unrestricted. However, the two phases have to be strongly correlated: for every $\varphi_{A_{0}}, \varphi_{\mu}$ can only vary in an interval $\Delta \varphi_{\mu} \lesssim 0.01$. Taking into account only the chargino contribution, one would obtain the restriction $\left|\varphi_{\mu}\right| \lesssim 0.01$.

In Fig. 4a we show the experimentally allowed regions for the eEDM and the nEDM, calculated in the Quark-Parton Model. For the paramters chosen and the measured spin densities of the proton [18] $\Delta_{u}=0.746, \Delta_{d}=-0.508$, and $\Delta_{s}=-0.226$, the allowed band in the $\varphi_{\mu}-\varphi_{A_{0}}$ plane of the nEDM lies within the allowed band of the eEDM. In this case the $n E D M$ is more restrictive. For the values of the spin densities taken, the nEDM and the eEDM have opposite signs. In Fig. $4 \mathrm{~b}$ we plot the allowed band in the $\varphi_{\mu}-\varphi_{A_{0}}$ plane of the nEDM, calculated in the Chiral Quark Model, and compare it to the eEDM. They have the same sign. As one can see, in this case only a very small region of the parameter space is not excluded by experiment: $\left|\varphi_{\mu}\right| \lesssim 0.01$ and $\left|\varphi_{A_{0}}\right| \lesssim 0.15$. (All phases have to be understood modulo $\pi$.)

In Figs. 5a and 5b we demonstrate the cancellation effects that play an essential role in the calculation of the nEDM. We choose the relation $\varphi_{\mu}=-(\pi / 30) \cdot \sin \varphi_{A_{0}}$, which guarantees that the nEDM calculated in the Quark-Parton Model fulfills the experimental bound. We show the different contributions to the nEDM for the same parameters as in Fig. 4a. In Fig. 5a we show the corresponding chargino, neutralino, and gluino contributions. As can be seen, there is a strong cancellation between chargino and gluino contributions: each of the two contributions is approximately 18 times bigger than the whole nEDM. In Fig. 5b we show the up, down, and strange quark contributions to the nEDM. Again, cancellations between the individual quark contributions occur. It turns out, that the strange quark contribution is the most important one, as noted in [12]. Therefore, it may turn out that an accurate measurement of the nEDM can also become a test of the spin structure of the neutron in the Quark-Parton Model.

In the Chiral Quark Model the cancellations occur for up and down quark seperately. There are large cancellations between $d_{\tilde{\chi}^{+}}^{n}$ and $d_{\tilde{g}}^{n}$, between $\hat{d}_{\tilde{\chi}^{+}}^{n}$ and $\hat{d}_{\tilde{g}}^{n}$, and also between the resulting sums of this cancellations (see Eq. (2.32)). The purely gluonic dimension-six operator does not exceed the experimental limit by itself, however, it can further reduce the total nEDM. For the eEDM the cancellation between chargino and neutralino contribution 
exhibits the same behaviour as shown in Fig. 5a, where the neutralino contribution in the eEDM plays the same role as the gluino contribution in the nEDM.

In Figs. 6a and $6 \mathrm{~b}$ we show the regions in the $M_{0^{-}} M_{1 / 2}$ plane which are excluded by simultanous consideration of the experimental limits on eEDM and nEDM. Fig. 6a is for the Quark-Parton Model and Fig. 6b is for the Chiral Quark Model. In both plots we choose the following values for the phases: $\varphi_{A_{0}}=-\pi / 10$ (dashed lines), $\varphi_{A_{0}}=\pi / 5$ (dotted lines), $\varphi_{A_{0}}=\pi / 2$ (dashed-dotted lines), $\varphi_{\mu}=-\pi / 10$ (thin lines), and $\varphi_{\mu}=-\pi / 30$ (thick lines). Only values of $\left(M_{1 / 2}, M_{0}\right)$ to the right of the corresponding lines are allowed by eEDM and nEDM simultanously. In Fig. 6a there are only four lines, because for $\varphi_{\mu}=-\pi / 10$, $\varphi_{A_{0}}=-\pi / 10$ and $\varphi_{\mu}=-\pi / 10, \varphi_{A_{0}}=\pi / 5$ the parameter region $M_{0}, M_{1 / 2} \lesssim 1.5 \mathrm{TeV}$ is excluded by experiment. The Quark-Parton Model is in general more restrictive than the Chiral Quark Model. However, in the Quark-Parton Model much smaller pairs of mass parameters are allowed, for example $M_{0}=150 \mathrm{GeV}$ and $M_{1 / 2}=200 \mathrm{GeV}$. As can be seen, the strongest cancellation effects are found for $\left|\varphi_{A_{0}}\right|=\pi / 2$ and $\operatorname{sign} \varphi_{A_{0}}=-\operatorname{sign} \varphi_{\mu}$. This is also observed in [11]. If $\varphi_{A_{0}}$ and $\varphi_{\mu}$ have the same sign, the exclusion is more or less indepentent of $M_{0}$.

Our numerical investigation of the $\mathrm{nEDM}$ includes the contributions of the one-loop gluino, chargino and neutralino exchange diagrams for the electric dipole operators. In the Chiral Quark Model we also include the chromoelectric dipole operators and the contribution of the purely gluonic dimension-six operator. In the following we want to discuss qualitatively which contributions are important to understand the behavior of the $\mathrm{nEDM}$ and its dependence on the mSUGRA parameters.

The dominant contributions to the nEDM come from the chargino and gluino exchange diagrams of the quark EDMs. It is remarkable that the chargino contribution is almost independent of the phase $\varphi_{A_{0}}$. This is due to the fact that the second terms of Eqs. (2.16) and (2.17) are suppressed by the Yukawa couplings $Y_{u, d}$ which are very small for light quarks. The gluino contribution, Eq. (2.20), depends on both phases, $\varphi_{\mu}$ and $\varphi_{A_{0}}$, since it is proportional to the off-diagonal element of the squark mass matrix, $m_{q}\left(A_{q}-\mu^{*} \Theta(\beta)\right)$, (see Eqs. (A1) and (A5)). The neutralino contributions to the quark EDMs are very small in contrast to the eEDM.

In the Chiral Quark Model the down quark contribution is the most important one, because the EDM is proportional to $\left(4 d_{d}-d_{u}\right)$. Moreover, for the chargino contribution we have $Y_{d}=\left(m_{d} / m_{u}\right) \tan \beta Y_{u} \gtrsim 6 Y_{u}$ if $\tan \beta \gtrsim 3$. The gluino contribution to the down quark is proportional to $m_{d} \operatorname{Im}\left[A_{d}-\mu^{*} \tan \beta\right]$, whereas the up quark EDM contains the factor $m_{u} \operatorname{Im}\left[A_{u}-\mu^{*} \cot \beta\right]$. Taking into account that $|\mu|$ and $\left|A_{q}\right|$ have the same order of magnitude, we make the following observations: The down quark EDM depends mainly on $\mu$. The up quark EDM is dominated by the term proportional to $A_{u}$ and is suppressed by a factor $\left(m_{u} / m_{d}\right) \cot \beta$ compared to the down quark term.

The chromoelectric contributions (see Sec. IID) are suppressed by a factor $g_{s} /(4 \pi)$ compared to the electric dipole operator and, in general, they are less important. In the case where $M_{0}>M_{1 / 2}$ the loop function $C$, Eq. (2.25), entering $\hat{d}_{\tilde{g}}^{q}$, Eq. (2.24), can compensate this suppression factor $g_{s} /(4 \pi)$. It also turns out that the contribution of the purely gluonic dimension-six operator is very small in the parameter region considered.

In order to see how the restrictions on $\varphi_{\mu}$ and $\varphi_{A_{0}}$ depend on the other parameters we also discuss a scenario with $\left|A_{0}\right|=M_{0}$. In Fig. 7 we show regions in the $\varphi_{\mu}-\varphi_{A_{0}}$ plane, 
allowed by the experimental bounds on eEDM and nEDM in this case. We calculate the nEDM in the Quark-Parton Model with $\left|A_{0}\right|=M_{0}=150 \mathrm{GeV}$ and the other parameters as in Fig. 4a. We find that the phase $\varphi_{A_{0}}$ is less important than in the previous scenario $\left(\left|A_{0}\right|=3 M_{0}\right)$. The allowed values of $\varphi_{\mu}$ are reduced roughly by a factor $1 / 3$ compared to Fig. 4a, thereby suggesting a linear dependence of the allowed values on $\left|A_{0}\right|$ keeping the other parameters fixed. Furthermore, the value of $\tan \beta$ effects the results in a similar way, because it enters in the off-diagonal element of the sfermion mixing matrix, Eqs. (A1)-(A6). This element is only important for the gluino contribution to the $\mathrm{nEDM}$ and the neutralino contribution to the eEDM. As can be seen in Fig. 7, the bands in the $\varphi_{\mu}-\varphi_{A_{0}}$ plane, allowed by the eEDM and the nEDM in the Quark-Parton Model, overlap similarly as in Fig. 4a.

In order to study the restrictions imposed by the universality conditions at the GUT scale, we modify the universality condition for the gaugino mass parameters, Eq. (3.2). We still assume $M_{1 / 2}:=M_{1}=M_{2}=\left|M_{3}\right|$, but introduce an additional phase $\varphi_{3}$ for the mass parameter $M_{3}$ at the GUT scale. We show in Fig. 8 the bands in the $\varphi_{\mu}-\varphi_{A_{0}}$ plane, allowed by the eEDM and the nEDM in the Quark-Parton Model, where we take for $\varphi_{3}$ the values 0 , $\pi / 10$, and $\pi / 5$. We take the other parameters as in Fig. 4a. The eEDM depends on $\varphi_{3}$ only via the RGEs, therefore, this dependence is very weak. Comparing the band of the eEDM (dotted line) with the bands of the $\mathrm{nEDM}$ for values of $\varphi_{3}$ different from zero, one can see that $\varphi_{3}$ is strongly restricted by experiment. A further possibility would be to introduce an additional phase $\varphi_{1}$ for the $U(1)$ gaugino mass parameter $M_{1}$. This phase will enter the eEDM and the nEDM. It is expected that $\varphi_{1}$ will change the restrictions on $\varphi_{3}$ in a similar way as the phase $\varphi_{A_{0}}$ changes the restrictions on $\varphi_{\mu}$.

\section{SUMMARY}

We have studied the eEDM and the nEDM in the framework of mSUGRA with complex parameters. We have found that $\varphi_{\mu}$ is strongly restricted by the experimental bounds. Moreover, we have found that the phases $\varphi_{\mu}$ and $\varphi_{A_{0}}$ have to be strongly correlated, in particular for small values of the SUSY mass parameters, so that strong cancellations between the different contributions occur. For the experimentally allowed values of the eEDM, the chargino contribution has to be cancelled by the neutralino contribution. The nEDM is dominated by the chargino and gluino contributions. The predictions for the nEDM depend very sensitively on the model which is used for the neutron. We have used the Quark-Parton Model and the Chiral Quark Model to calculate the nEDM. We have presented parameter regions in the $M_{0}-M_{1 / 2}$ plane which are excluded by simultanous consideration of the experimental bounds on the eEDM and the nEDM for different values of the phases $\varphi_{\mu}$ and $\varphi_{A_{0}}$.

\section{ACKNOWLEDGEMENTS}

This work has been supported by the 'Fonds zur Förderung der wissenschaftlichen Forschung' of Austria, projects no. P10843-PHY and no. P13139-PHY, and by 'Acciones Integradas'. We are grateful to E. Christova, J. Ellis, W. Grimus, and W. Majerotto for very useful discussions. 


\section{APPENDIX A: SFERMION MASS MATRIX}

The sfermion mass matrices are given by

$$
M_{\tilde{f}}^{2}=\left(\begin{array}{cc}
M_{\tilde{f}_{L L}}^{2} & e^{-i \varphi_{\tilde{f}}} M_{\tilde{f}_{L R}}^{2} \\
e^{i \varphi_{\tilde{f}}} M_{\tilde{f}_{L R}}^{2} & M_{\tilde{f}_{R R}}^{2}
\end{array}\right)
$$

where

$$
\begin{aligned}
M_{\tilde{f}_{L L}}^{2} & =M_{L \tilde{f}}^{2}+\left(T_{I}^{3}-Q_{f} \sin ^{2} \theta_{W}\right) \cos 2 \beta m_{Z}^{2}+m_{f}^{2}, \\
M_{\tilde{f}_{R R}}^{2} & =M_{R \tilde{f}}^{2}+Q_{f} \sin ^{2} \theta_{W} \cos 2 \beta m_{Z}^{2}+m_{f}^{2}, \\
M_{\tilde{f}_{L R}}^{2} & =m_{f}\left|A_{f}-\mu^{*} \Theta(\beta)\right|, \\
\varphi_{\tilde{f}} & =\arg \left[A_{f}-\mu^{*} \Theta(\beta)\right],
\end{aligned}
$$

with

$$
\Theta(\beta)=\left\{\begin{array}{ll}
\cot \beta & \text { for } T_{I}^{3}=\frac{1}{2} \\
\tan \beta & \text { for } T_{I}^{3}=-\frac{1}{2}
\end{array} .\right.
$$

The eigenvalues are given by

$$
2 m_{1,2}^{2}=\left(M_{\tilde{f}_{L L}}^{2}+M_{\tilde{f}_{R R}}^{2}\right) \mp \sqrt{\left(M_{\tilde{f}_{L L}}^{2}-M_{\tilde{f}_{R R}}^{2}\right)^{2}+4\left(M_{\tilde{f}_{L R}}^{2}\right)^{2}},
$$

with $m_{1}^{2} \leq m_{2}^{2}$. We parametrize the mixing matrix $\mathcal{R}^{\tilde{f}}$ so that

$$
\left(\begin{array}{c}
\tilde{f}_{1} \\
\tilde{f}_{2}
\end{array}\right)=\mathcal{R}^{\tilde{f}}\left(\begin{array}{c}
\tilde{f}_{L} \\
\tilde{f}_{R}
\end{array}\right)=\left(\begin{array}{cc}
e^{\frac{i}{2} \varphi_{\tilde{f}}} \cos \theta_{\tilde{f}} & e^{-\frac{i}{2} \varphi_{\tilde{f}}} \sin \theta_{\tilde{f}} \\
-e^{\frac{i}{2} \varphi_{\tilde{f}}} \sin \theta_{\tilde{f}} & e^{-\frac{i}{2} \varphi_{\tilde{f}}} \cos \theta_{\tilde{f}}
\end{array}\right)\left(\begin{array}{c}
\tilde{f}_{L} \\
\tilde{f}_{R}
\end{array}\right)
$$

where $\varphi_{\tilde{f}}$ is given in Eq. (A5) and

$$
\begin{aligned}
& \cos \theta_{\tilde{f}}=\frac{-M_{\tilde{f}_{L R}}^{2}}{\Delta} \leq 0, \quad \sin \theta_{\tilde{f}}=\frac{M_{\tilde{f}_{L L}}^{2}-m_{1}^{2}}{\Delta} \geq 0 \\
& \Delta^{2}=\left(M_{\tilde{f}_{L R}}^{2}\right)^{2}+\left(m_{1}^{2}-M_{\tilde{f}_{L L}}^{2}\right)^{2}
\end{aligned}
$$

\section{APPENDIX B: CHARGINO MASS MATRIX}

The chargino mass matrix

$$
M_{\alpha \beta}^{\tilde{\chi}^{+}}=\left(\begin{array}{cc}
M & m_{W} \sqrt{2} \sin \beta \\
m_{W} \sqrt{2} \cos \beta & \mu
\end{array}\right)
$$

can be diagonalized by the biunitary transformation

$$
U_{j \alpha}^{*} M_{\alpha \beta}^{\tilde{\chi}^{+}} V_{k \beta}^{*}=m_{\tilde{\chi}_{j}^{+}} \delta_{j k}
$$

where $U$ and $V$ are unitary matrices such that $m_{\tilde{\chi}_{j}^{+}}$are positiv and $m_{\tilde{\chi}_{1}^{+}}<m_{\tilde{\chi}_{2}^{+}}$. 


\section{APPENDIX C: NEUTRALINO MASS MATRIX}

We define $N_{\alpha j}$ as the unitary matrix which makes the complex symmetric neutralino mass matrix diagonal with positiv diagonal elements:

$$
N_{\alpha j} M_{\alpha \beta}^{\tilde{\chi}^{0}} N_{\beta k}=m_{\tilde{\chi}_{j}} \delta_{j k},
$$

where $m_{\tilde{\chi}_{j}^{0}}<m_{\tilde{\chi}_{k}^{0}}$ for $j<k$. In the basis [24]:

$$
\psi_{\alpha}=\left\{-i \tilde{\gamma},-i \tilde{Z}, \tilde{H}^{a}, \tilde{H}^{b}\right\},
$$

the complex symmetric neutralino mass matrix has the form

$$
M_{\alpha \beta}^{\tilde{\chi}^{0}}=\left(\begin{array}{cccc}
m_{\tilde{\gamma}} & m_{a z} & 0 & 0 \\
m_{a z} & m_{\tilde{z}} & m_{Z} & 0 \\
0 & m_{Z} & \mu \sin 2 \beta & -\mu \cos 2 \beta \\
0 & 0 & -\mu \cos 2 \beta & -\mu \sin 2 \beta
\end{array}\right),
$$

where

$$
\begin{aligned}
m_{\tilde{\gamma}} & =M \sin ^{2} \theta_{W}+M^{\prime} \cos ^{2} \theta_{W}, \\
m_{\tilde{z}} & =M \cos ^{2} \theta_{W}+M^{\prime} \sin ^{2} \theta_{W}, \\
m_{a z} & =\sin \theta_{W} \cos \theta_{W}\left(M-M^{\prime}\right) .
\end{aligned}
$$

\section{APPENDIX D: SOLUTIONS OF THE ONE-LOOP RGES}

The solutions of the one-loop RGEs (as given in [20]) for the soft SUSY-breaking parameters are given by

$$
\begin{aligned}
M_{i}(t) & =\frac{M_{1 / 2}}{1+\beta_{i} t} \\
M_{\tilde{E}_{1}}^{2}(t) & =M_{0}^{2}+\frac{\alpha_{G U T} M_{1 / 2}^{2}}{4 \pi} \frac{6}{5} f_{1}(t) \\
M_{\tilde{L}_{1}}^{2}(t) & =M_{0}^{2}+\frac{\alpha_{G U T} M_{1 / 2}^{2}}{4 \pi}\left(\frac{3}{2} f_{2}(t)+\frac{3}{10} f_{1}(t)\right) \\
M_{\tilde{D}_{1}}^{2}(t) & =M_{0}^{2}+\frac{\alpha_{G U T} M_{1 / 2}^{2}}{4 \pi}\left(\frac{8}{3} f_{3}(t)+\frac{2}{15} f_{1}(t)\right) \\
M_{\tilde{U}_{1}}^{2}(t) & =M_{0}^{2}+\frac{\alpha_{G U T} M_{1 / 2}^{2}}{4 \pi}\left(\frac{8}{3} f_{3}(t)+\frac{8}{15} f_{1}(t)\right) \\
M_{\tilde{Q}_{1}}^{2}(t) & =M_{0}^{2}+\frac{\alpha_{G U T} M_{1 / 2}^{2}}{4 \pi}\left(\frac{8}{3} f_{3}(t)+\frac{3}{2} f_{2}(t)+\frac{1}{30} f_{1}(t)\right) \\
A_{t}(t) & =\frac{A_{0}}{1+6 Y_{t}(0) F(t)}-M_{1 / 2}\left(H_{1}(t)-\frac{6 Y_{t}(0) H_{2}(t)}{1+6 Y_{t}(0) F(t)}\right)
\end{aligned}
$$




$$
\begin{aligned}
& A_{u}(t)=\frac{1}{2}\left(A_{0}+A_{t}(t)-M_{1 / 2} H_{1}(t)\right) \\
& A_{d}(t)=A_{0}-\frac{\alpha_{G U T} M_{1 / 2}}{4 \pi}\left(\frac{16}{3} j_{3}(t)+3 j_{2}(t)+\frac{7}{15} j_{1}(t)\right) \\
& A_{e}(t)=A_{0}-\frac{\alpha_{G U T} M_{1 / 2}}{4 \pi}\left(3 j_{2}(t)+\frac{9}{5} j_{1}(t)\right)
\end{aligned}
$$

with

$$
\begin{aligned}
t & =\ln \left(M_{G U T} / Q\right)^{2} \\
\beta_{i} & =\frac{\alpha_{G U T}}{4 \pi} b_{i} \\
f_{i}(t) & =\frac{1}{\beta_{i}}\left(1-\frac{1}{\left(1+\beta_{i} t\right)^{2}}\right) \\
j_{i}(t) & =\frac{t}{1+\beta_{i} t} \\
Y_{t}(t) & =\frac{h_{t}^{2}(t)}{(4 \pi)^{2}} \\
E(t) & =\left(1+\beta_{3} t\right)^{\frac{16}{3 b_{3}}}\left(1+\beta_{2} t\right)^{\frac{3}{b_{2}}}\left(1+\beta_{1} t\right)^{\frac{13}{9 b_{1}}} \\
F(t) & =\int_{0}^{t} E(s) d s \\
H_{1}(t) & =\frac{\alpha_{G U T}}{4 \pi}\left(\frac{16}{3} j_{3}(t)+3 j_{2}(t)+\frac{13}{15} j_{1}(t)\right) \\
H_{2}(t) & =t E(t)-F(t)
\end{aligned}
$$

where $b_{1}=11, b_{2}=1$, and $b_{3}=-3$. Some of the equations can be found in 25. 


\section{REFERENCES}

[1] I.S. Altarev et al., Phys. Lett. B 276, 242 (1992); E. Commins et al., Phys. Rev. A 50, 2960 (1994); Euro. Phys. Jour. C 3, 1 (1998).

[2] For a review, see: S.M. Barr and W.J. Marciano, in "CP-Violation", ed. C. Jarlskog (World Scientific, Singapore 1989), p. 455; W. Bernreuther and M. Suzuki, Rev. Mod. Phys. 63, 313 (1991).

[3] J. Ellis, S. Ferrara, and D.V. Nanopoulos, Phys. Lett. 114B, 231 (1982); W. Buchmüller and D. Wyler, Phys. Lett. 121B, 321 (1983); J. Polchinski and M.B. Wise, Phys. Lett. 125B, 393 (1983); J.M. Gerard, W. Grimus, A. Masiero, D.V. Nanopoulos, and A. Raychaudhuri, Nucl. Phys. B253, 93 (1985).

[4] P. Nath, Phys. Rev. Lett. 66, 2565 (1991); Y. Kizukuri and N. Oshimo, Phys. Rev. D 45, 1806 (1992); Phys. Rev. D 46, 3025 (1992).

[5] M. Carena, M. Quirós, and C.E.M. Wagner, Nucl. Phys. B524, 3 (1998); for a review, see: A.G. Cohen, D.B. Kaplan, and A.E. Nelson, Ann. Rev. Nucl. Part. Sci. 43, 27 (1993).

[6] M. Drees and S.P. Martin, in "Electroweak Symmetry Breaking and New Physics at the TeV Scale", ed. T.L. Barklow et al., (World Scientific, Singapore 1997).

[7] R. Garisto and J.D. Wells, Phys. Rev. D 55, 1611 (1997).

[8] T. Falk, K.A. Olive, and M. Srednicki, Phys. Lett. B 354, 99 (1995).

[9] T. Ibrahim and P. Nath, Phys. Lett. B 418, 98 (1998); T. Ibrahim and P. Nath, Phys. Rev. D 57, 478 (1998); T. Ibrahim and P. Nath, Phys. Rev. D 58, 111301 (1998).

[10] T. Falk and K.A. Olive, Phys. Lett. B 375, 196 (1996); Phys. Lett. B 439, 71 (1998).

[11] M. Brhlik, G.J. Good, and G.L. Kane, hep-ph / 9810457, UM-TH-98-16.

[12] J. Ellis and R.A. Flores, Phys. Lett. B 377, 83 (1996).

[13] A. Manohar and H. Georgi, Nucl. Phys. B234, 189 (1984).

[14] S. Kraml, H. Eberl, A. Bartl, W. Majerotto, and W. Porod, Phys. Lett. B 386, 175 (1996).

[15] G. Ecker, W. Grimus, and H. Neufeld, Nucl. Phys. B229, 421 (1983);

[16] A. Bartl, E. Christova, T. Gajdosik, and W. Majerotto, Nucl. Phys. B507, 35 (1997); Nucl. Phys. B531, 653(E) (1998); W. Hollik, J.I. Illana, S. Rigolin, and D. Stöckinger, Phys. Lett. B 425, 322 (1998); W. Hollik, J.I. Illana, S. Rigolin, C. Schappacher, and D. Stöckinger, hep-ph / 9808408, KA-TP-10-1998.

[17] J. Dai, H. Dykstra, R.G. Leigh, and S. Paban, Phys. Lett. B 237, 216 (1990).

[18] J. Ashman et al., European Muon Collaboration, Phys. Lett. B 206, 364 (1988); Nucl. Phys. B328, 1 (1989).

[19] R. Arnowitt, J. Lopez, and D.V. Nanopoulos, Phys. Rev. D 42, 2423 (1990); R. Arnowitt, M. Duff, and K. Stelle, Phys. Rev. D 43, 3085 (1991).

[20] D.J. Castaño, E.J. Piard, and P. Ramond, Phys. Rev. D 49, 4908 (1994).

[21] V. Barger, M.S. Berger, and P. Ohmann, Phys. Rev. D 49, 4908 (1994).

[22] H. Eberl and W. Majerotto, HEPHY-PUB 602/94.

[23] P. Martin and M.T. Vaughn, Phys. Rev. D 50, 2282 (1994); Y. Yamada, Phys. Rev. D 50, 3537 (1994)

[24] A. Bartl, H. Fraas, W. Majerotto, and N. Oshimo, Phys. Rev. D 40, 1594 (1989) .

[25] L.E. Ibáñez and C. López, Nucl. Phys. B233, 511 (1984); L.E. Ibáñez and J. Mas, Nucl. Phys. B286, 107 (1987). 


\section{FIGURES}

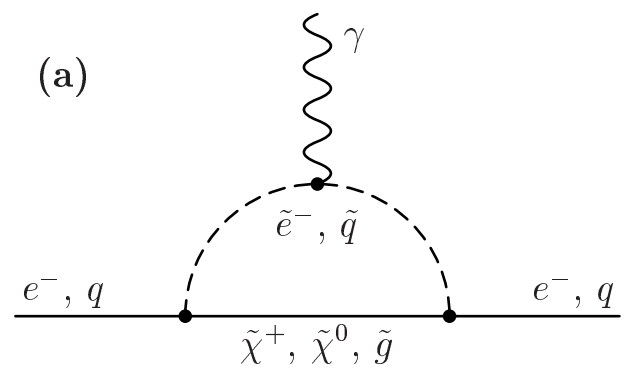

(b)

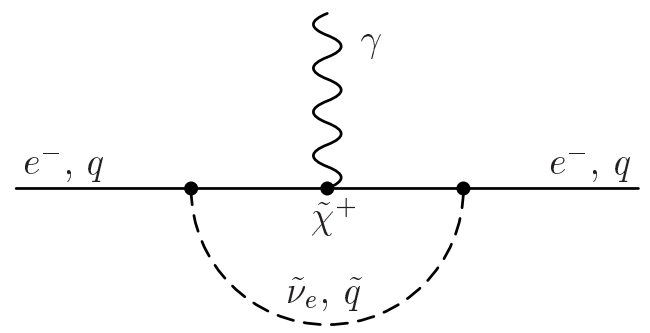

FIG. 1. The Feynman diagrams that contribute to the EDMs.

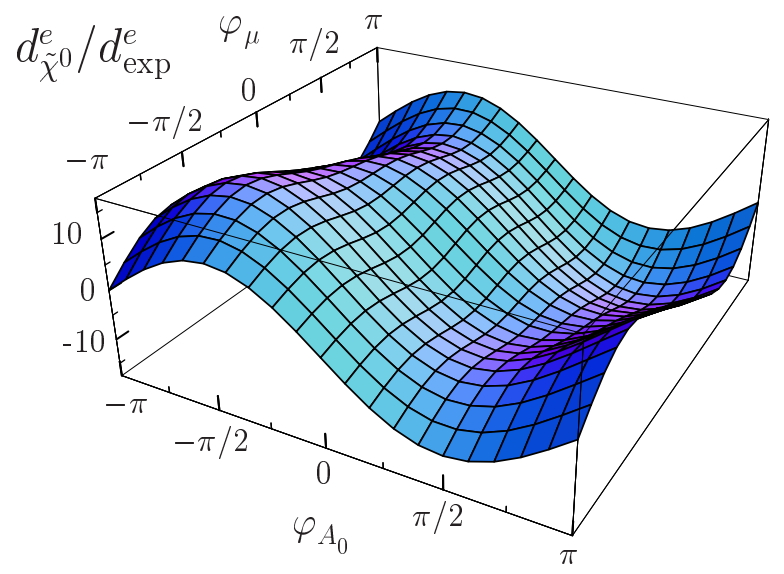

FIG. 2. Ratio of the neutralino contribution $d_{\tilde{\chi}^{0}}^{e}$ and the experimental limit $d_{\exp }^{e}$ of the electron EDM as a function of the phases $\varphi_{\mu}$ and $\varphi_{A_{0}}$. The mSUGRA parameters are $M_{0}=150 \mathrm{GeV}$, $M_{1 / 2}=200 \mathrm{GeV},\left|A_{0}\right|=450 \mathrm{GeV}$, and $\tan \beta=3$. 


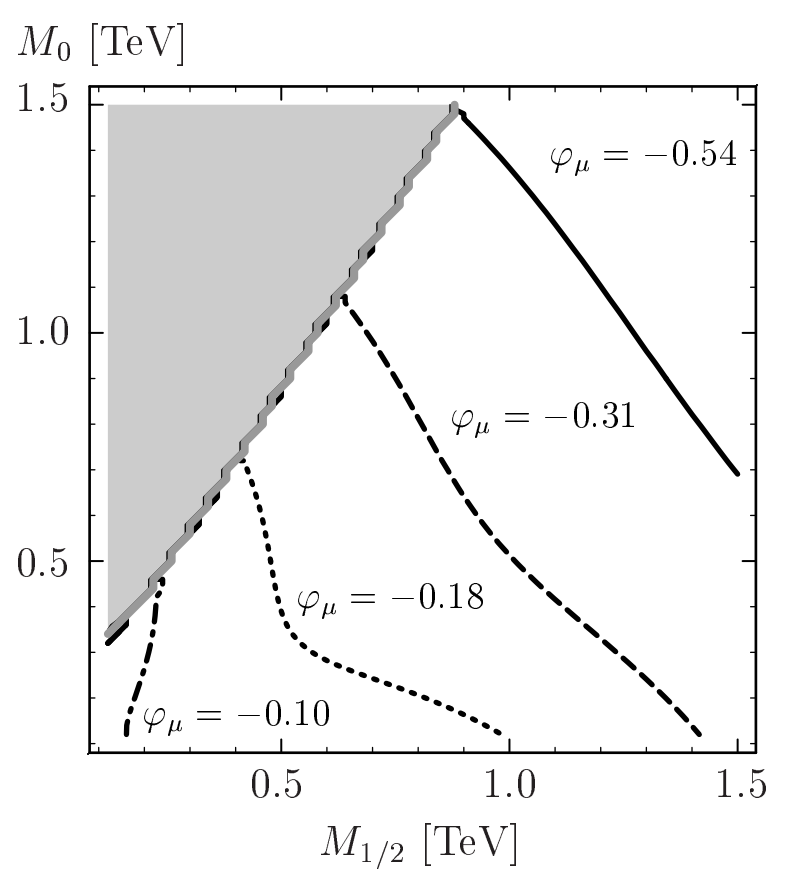

FIG. 3. Boundaries of the areas in the $M_{0}-M_{1 / 2}$ plane excluded by the electron EDM, for the phases $\varphi_{A_{0}}=\pi / 2$, and $\varphi_{\mu}=-0.54$ (solid line), $\varphi_{\mu}=-0.31$ (dashed line), $\varphi_{\mu}=-0.18$ (dotted line), $\varphi_{\mu}=-0.1$ (dashed-dotted line). The areas to the left of the corresponding lines are excluded. The mSUGRA parameters are $\left|A_{0}\right|=3 M_{0}$ and $\tan \beta=3$. In the grey area the condition of radiative electroweak symmetry breaking is not fulfilled.

Quark-Parton Model

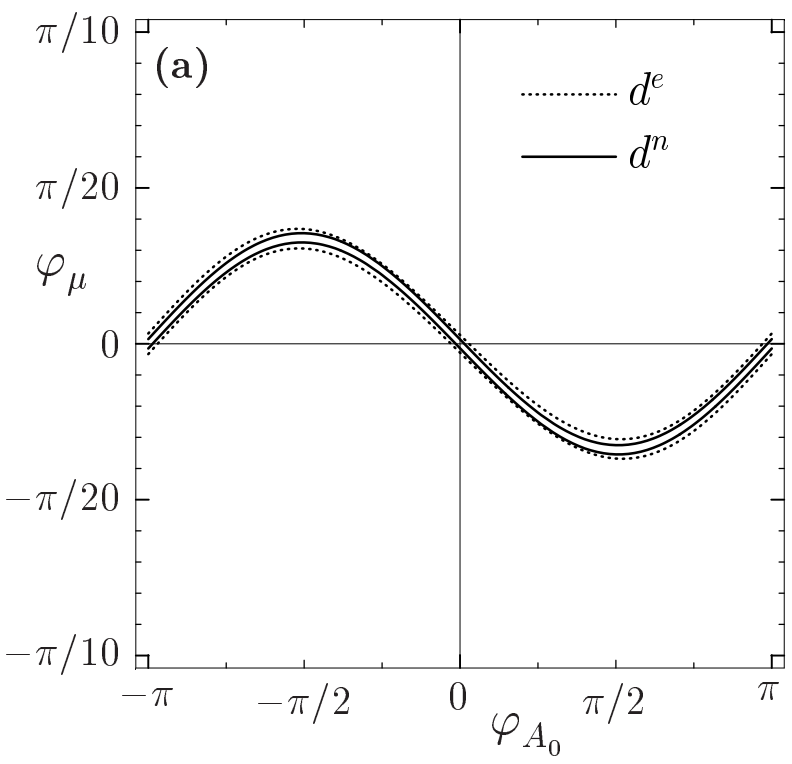

Chiral Quark Model

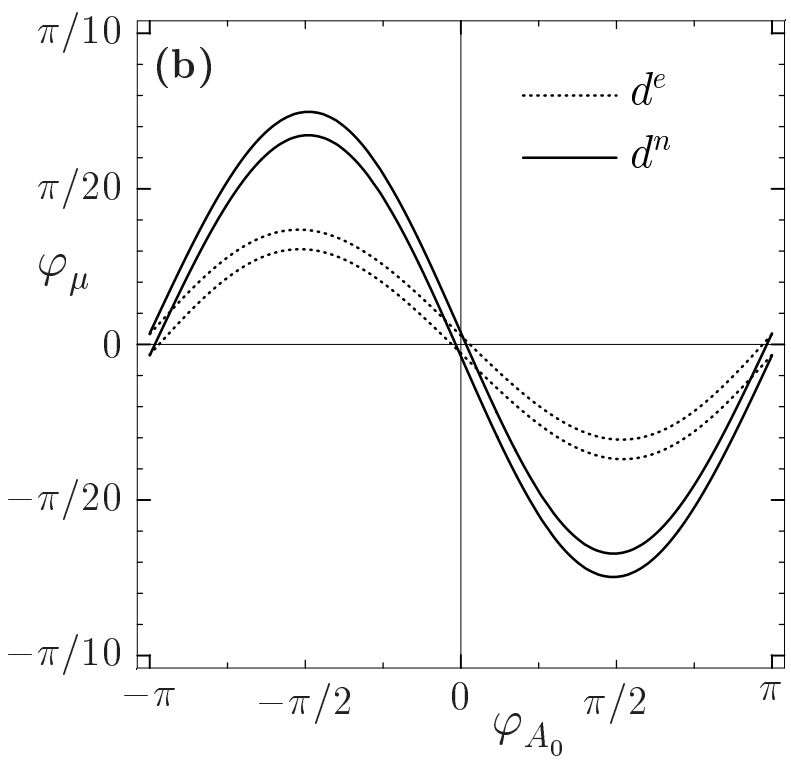

FIG. 4. Bands in the $\varphi_{\mu}-\varphi_{A_{0}}$ plane allowed by the electron EDM (dotted line) and neutron EDM (solid line). The mSUGRA parameters are $M_{0}=150 \mathrm{GeV}, M_{1 / 2}=200 \mathrm{GeV},\left|A_{0}\right|=450 \mathrm{GeV}$, and $\tan \beta=3$. The neutron EDM is calculated in the Quark-Parton Model (a) and in the Chiral Quark Model (b). 

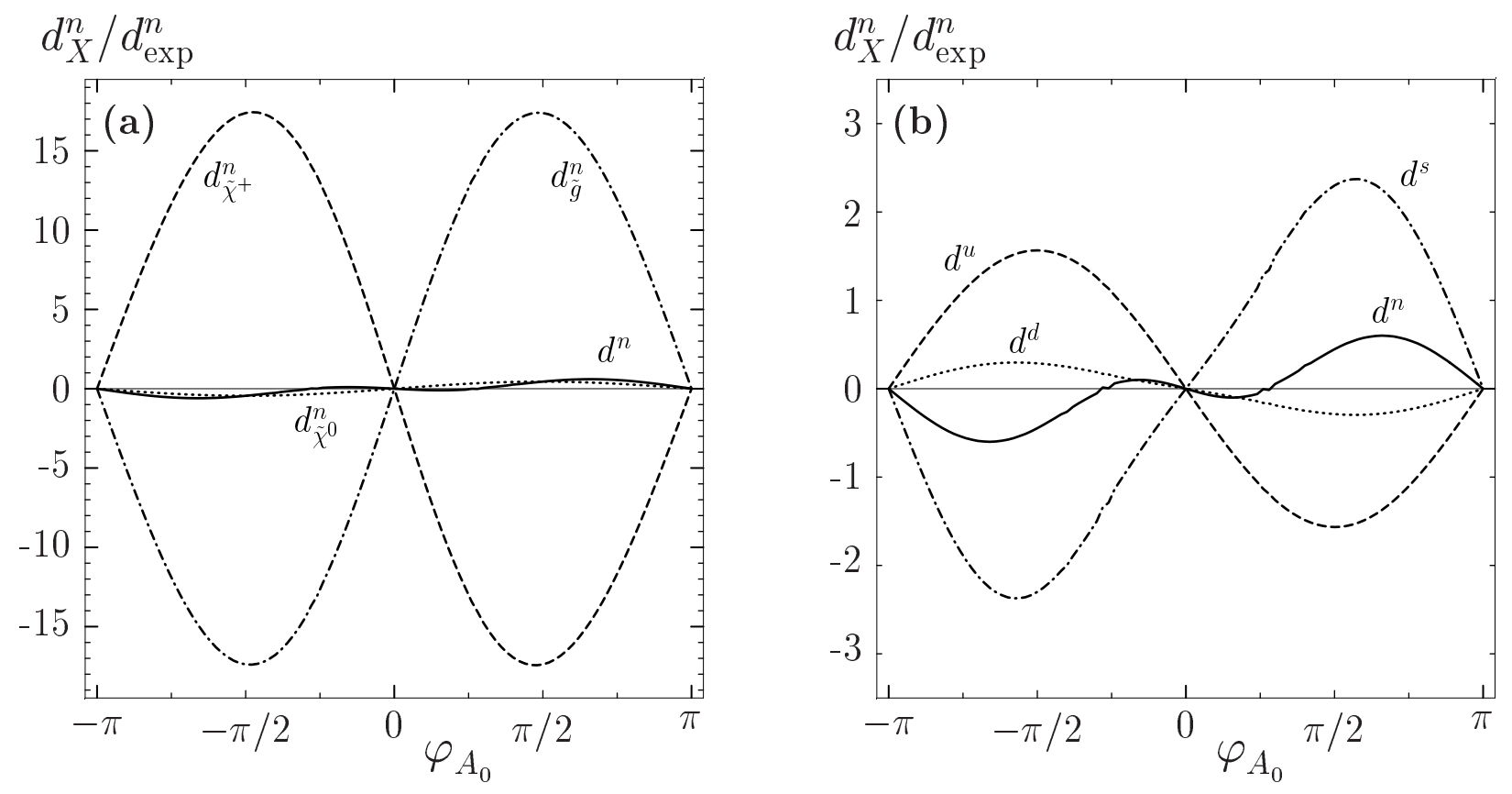

FIG. 5. Cancellations of the various contributions to the neutron EDM in the Quark-Parton Model, taking the relation $\varphi_{\mu}=-(\pi / 30) \cdot \sin \varphi_{A_{0}}$. The mSUGRA parameters are $M_{0}=150 \mathrm{GeV}$, $M_{1 / 2}=200 \mathrm{GeV},\left|A_{0}\right|=450 \mathrm{GeV}$, and $\tan \beta=3$. (a) shows the chargino contribution $d_{\tilde{\chi}^{+}}^{n}$ (dashed line), neutralino contribution $d_{\tilde{\chi}^{0}}^{n}$ (dotted line), gluino contribution $d_{\tilde{g}}^{n}$ (dashed-dotted line), and the whole neutron EDM $d^{n}$ (solid line). (b) shows the up quark contribution $d^{u}$ (dashed line), down quark contribution $d^{d}$ (dotted line), strange quark contribution $d^{s}$ (dashed-dotted line), and the whole neutron EDM $d^{n}$ (solid line). 

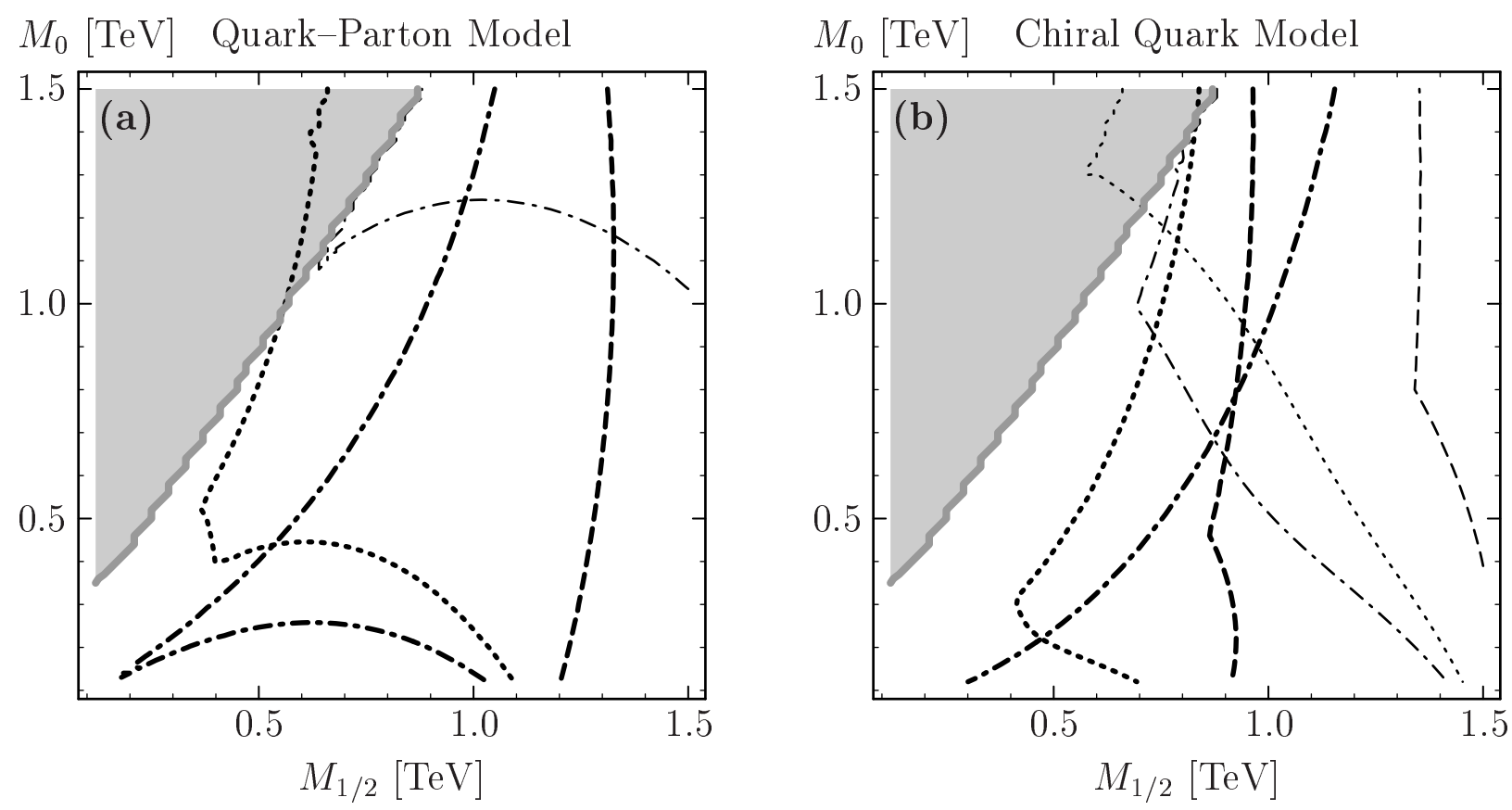

FIG. 6. Boundaries of the areas in the $M_{0}-M_{1 / 2}$ plane allowed simultanously by the electron EDM and the neutron EDM. The neutron EDM is calculated in the Quark-Parton Model (a) and in the Chiral Quark Model (b). The phases are chosen as $\varphi_{A_{0}}=-\pi / 10$ (dashed lines), $\varphi_{A_{0}}=\pi / 5$ (dotted lines), $\varphi_{A_{0}}=\pi / 2$ (dashed-dotted lines), and $\varphi_{\mu}=-\pi / 10$ (thin lines), $\varphi_{\mu}=-\pi / 30$ (thick lines). The areas to the left and below the corresponding lines are excluded. The mSUGRA parameters are $\left|A_{0}\right|=3 M_{0}$ and $\tan \beta=3$. In the grey area the condition of radiative electroweak symmetry breaking is not fulfilled. In (a) the whole parameter region is excluded for $\varphi_{\mu}=-\pi / 10$, $\varphi_{A_{0}}=-\pi / 10$ and $\varphi_{\mu}=-\pi / 10, \varphi_{A_{0}}=\pi / 5$. 


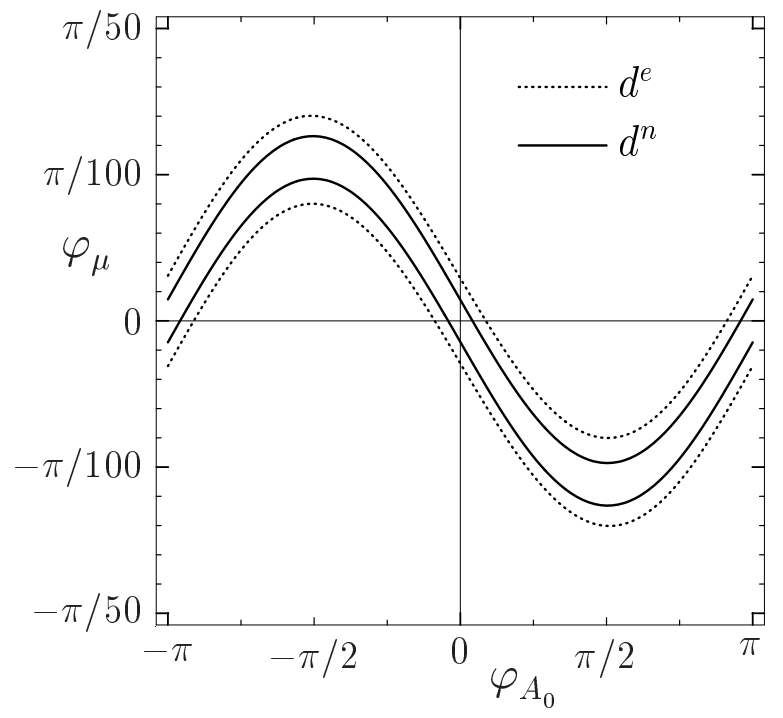

FIG. 7. Bands in the $\varphi_{\mu}-\varphi_{A_{0}}$ plane allowed by the electron EDM (dotted line) and neutron EDM (solid line). The mSUGRA parameters are $M_{0}=150 \mathrm{GeV}, M_{1 / 2}=200 \mathrm{GeV}$, $\left|A_{0}\right|=M_{0}=150 \mathrm{GeV}$, and $\tan \beta=3$. The neutron EDM is calculated in the Quark-Parton Model.

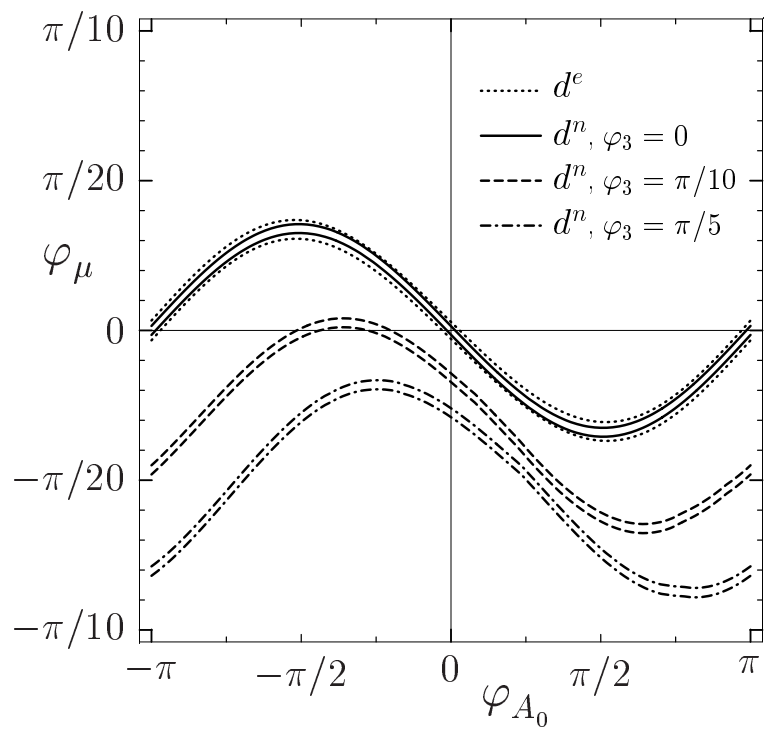

FIG. 8. Bands in the $\varphi_{\mu}-\varphi_{A_{0}}$ plane allowed by the electron EDM (dotted lines) and neutron EDM, calculated in the Quark-Parton Model, for $\varphi_{3}=0$ (solid lines), $\varphi_{3}=\pi / 10$ (dashed lines), and $\varphi_{3}=\pi / 5$ (dashed-dotted lines). The mSUGRA parameters are $M_{0}=150 \mathrm{GeV}, M_{1 / 2}=200 \mathrm{GeV}$, $\left|A_{0}\right|=450 \mathrm{GeV}$, and $\tan \beta=3$. 


\section{TABLES}

TABLE I. Phases occuring in the mass matrices at the electroweak scale and at the GUT scale.

\begin{tabular}{lll}
\hline \hline mass matrix & electroweak scale & GUT scale \\
\hline$M_{\tilde{u}}^{2}$ & $\varphi_{\tilde{u}}=\arg \left[A_{u}-\mu^{*} \cot \beta\right]$ & $\varphi_{A_{0}}, \varphi_{\mu}$ \\
$M_{\tilde{d}}^{2}$ & $\varphi_{\tilde{d}}=\arg \left[A_{d}-\mu^{*} \tan \beta\right]$ & $\varphi_{A_{0}}, \varphi_{\mu}$ \\
$M_{\tilde{e}}^{2}$ & $\varphi_{\tilde{e}}=\arg \left[A_{e}-\mu^{*} \tan \beta\right]$ & $\varphi_{A_{0}}, \varphi_{\mu}$ \\
$M^{\tilde{\chi}^{+}}$ & $\varphi_{\mu}$ & $\varphi_{\mu}$ \\
$M^{\tilde{\chi}^{0}}$ & $\varphi_{\mu}$ & $\varphi_{\mu}$ \\
\hline \hline
\end{tabular}

TABLE II. Values of the phases at the electroweak scale for $M_{1 / 2}$ real and $A_{0}=i A$ imaginary at the GUT scale when $A=x M_{1 / 2}$.

\begin{tabular}{lcccc}
\hline \hline $\mathrm{x}$ & $\left.\varphi_{A_{t}}\right|_{y=0.85(1)}$ & $\left.\varphi_{A_{u}}\right|_{y=0.85(1)}$ & $\varphi_{A_{d}}$ & $\varphi_{A_{e}}$ \\
\hline 0.1 & $-0.007(0)$ & $-0.021(-0.018)$ & -0.0278 & -0.142 \\
1 & $-0.075(0)$ & $-0.203(-0.177)$ & -0.271 & -0.960 \\
10 & $-0.644(0)$ & $-1.236(-1.190)$ & -1.207 & -1.501 \\
\hline \hline
\end{tabular}

